\title{
SOME EXAMPLES OF CYCLIC VECTORS IN THE DIRICHLET SPACE
}

\author{
LEON BROWN AND WILLIAM COHN ${ }^{1}$
}

\begin{abstract}
We consider the Hilbert space of analytic functions in the open unit disc that have a finite Dirichlet integral. For $E$, a closed subset of the unit circle with logarithmic capacity zero, we construct a function in this space which is uniformly continuous, vanishes on $E$, and is cyclic with respect to the shift operator.
\end{abstract}

For $\Delta$, the open unit disk in the complex plane, let $D$ be the space of functions $f$ analytic on $\Delta$ with finite Dirichlet integral

$$
\iint_{\Delta}\left|f^{\prime}\right|^{2} d x d y<\infty
$$

If $f$ has a Taylor expansion

$$
f(z)=\sum_{n=0}^{\infty} \hat{f}(n) z^{n}
$$

then

$$
\|f\|^{2} \equiv \sum_{n=0}^{\infty}(n+1)|\hat{f}(n)|^{2}
$$

defines a norm on $D$ which makes $D$ a Hilbert space. The problem of identifying the cyclic vectors in $D$, that is, those functions $f$ such that the set $\mathcal{P} f=\{p f: p$ a polynomial $\}$ is dense in $D$ was studied in $[\mathbf{1}]$. Denote by $[f]$ the closure of $P f$. The following result was established.

THEOREM A [1, THEOREM 5]. If $f$ is any function in $D$ whose radial limit vanishes on a set of positive logarithmic capacity then $f$ is not cyclic.

Recall that if $f \in D$ and $T$ is the unit circle then

$$
f^{*}\left(e^{i \theta}\right)=\lim _{r \rightarrow 1^{-}} f\left(r e^{i \theta}\right)
$$

exists for $e^{i \theta} \in T \backslash E$ where $E$ is a set of zero logarithmic capacity depending on $f$; see $[4$, Chapter IV, Théorème I] for a stronger result.

Question 12 of [1] asks if the converse of Theorem A holds for an outer function $f \in D$. That is, letting $Z(f)$ denote the zero set of $f^{*}$ for an outer function $f$, if $Z\left(f^{*}\right)$ has zero logarithmic capacity, must $f$ be cyclic? In fact, no examples were given in $[\mathbf{1}]$ of cyclic vectors $f$ for which $Z(f)$ was uncountable.

In this note, we modify a construction presented by Carleson (see the proof of Theorem 4 in [2]) to obtain the following result.

Received by the editors October 1, 1984.

1980 Mathematics Subject Classification. Primary 30H05; Secondary 46E20, 47B37.

Key words and phrases. Hilbert space of analytic functions, Dirichlet integral, cyclic vectors, logarithmic capacity.

${ }^{1}$ The research of both authors was supported in part by the National Science Foundation. 
THEOREM B. Let $E$ be a closed subset of the circle $T$ of zero logarithmic capacity. Then there exists a function $f \in D$ which is continuous on the closed disk $\bar{\Delta}$, cyclic in $D$ and $Z(f)=E$.

In the literature there are several definitions of logarithmic capacity. We choose to use the one presented in Chapter III of [3]. We prefer the kernel to be positive for $r=|z-w|, z, w \in \bar{\Delta}$, and set, following the notation of $[\mathbf{3}]$,

$$
H(t)= \begin{cases}t+\log 4, & t \geq-\log 4, \\ 0, & t<-\log 4,\end{cases}
$$

and

$$
K(r)=H(\log 1 / r)=\log ^{+}(4 / r) .
$$

It is a simple matter to verify that the sets of capacity zero obtained by using the kernel $K$ as in $[\mathbf{3}]$ are the same as the sets of logarithmic capacity zero obtained by other authors.

If $E$ is a bounded Borel set, let $\Gamma_{E}$ be the class of positive measures $\mu$ with support contained in $E$ such that $U_{\mu}(z) \leq 1, z \in E$, where $U_{\mu}$ is the logarithmic potential

$$
U_{\mu}(z)=\int K(|z-w|) d \mu(w)
$$

Also, define the energy integral $I(\mu)$,

$$
I(\mu)=\int U_{\mu}(z) d \mu(z)=\iint K(|z-w|) d \mu(w) d \mu(z) .
$$

The (logarithmic) capacity $C_{K}(E)$ of $E$ is defined by the relation

$$
\operatorname{cap}(E)=C_{K}(E)=\sup _{\mu \in \Gamma_{E}} \mu(E)=\sup _{\mu \in \Gamma_{E}}\|\mu\| .
$$

If $E$ is compact there exists a unique measure $\sigma$ in $\Gamma_{E}$ such that $\|\sigma\|=\operatorname{cap}(E)$; see Theorem 6 , p. 20 in [3]. The potential $U_{\sigma}$ is called the conductor potential for $E$.

With this notation and theory established we are ready to begin the proof of Theorem B.

If $E$ is a compact subset of $T$ and $\operatorname{cap}(E)=0$ we may choose closed subsets $I_{n}$ of $T$ each consisting of finitely many closed arcs such that

(i) $E \subseteq I_{n}, n=1,2, \ldots$,

(ii) $\sum_{n=1}^{\infty} \operatorname{cap}\left(I_{n}\right)^{1 / 2}<\infty$,

(iii) $\bigcap_{n=1}^{\infty} I_{n}=E$.

Let $U_{n}$ be the conductor potential for $I_{n}$ and $\sigma_{n}$ the unique measure defining this potential. We claim that

(1) $U_{n}=1$ p.p. on $I_{n}$, i.e., $U_{n}=1$ on $I_{n}$ except possibly on a set of capacity zero;

(2) $0<U_{n} \leq 1$

(3) $\left\|\sigma_{n}\right\|=\operatorname{cap}\left(I_{n}\right)$.

These three claims follow easily from $[3$, Theorem 4, p. 19 and Theorem 1, p. 15] and the definition of $\sigma_{n}$. We also claim that

(4) $\hat{\sigma}_{n}(0)^{2} \log ^{2} 4+\sum_{k=1}^{\infty}\left|\hat{\sigma}_{n}(k)\right|^{2} / k \leq \operatorname{cap}\left(I_{n}\right)$, where

$$
\hat{\sigma}_{n}(k)=\frac{1}{2 \pi} \int_{-\pi}^{\pi} e^{-i k \theta} d \sigma_{n}(\theta) .
$$


For this, from (2) and (3) we have

$$
I\left(\sigma_{n}\right) \leq \int U_{n}(z) d \sigma_{n}(z) \leq \sigma_{n}\left(I_{n}\right)=\left\|\sigma_{n}\right\|=\operatorname{cap}\left(I_{n}\right) .
$$

By [4, Proposition 3, p. 35] we have

$$
I\left(\sigma_{n}\right)=\hat{\sigma}_{n}(0)^{2} \log ^{2} 4+\sum \frac{\left|\hat{\sigma}_{n}(k)\right|^{2}}{k} .
$$

See the proof of $[\mathbf{1}$, Theorem 5] for details. This proves (4).

By representing $U_{n}$ as the Poisson Integral of $U_{n}\left(e^{i \theta}\right)$ and using properties (1) and (2) above, we see that for each $n$ we may choose $0<r_{n}<1$ so close to 1 that

$$
U_{n}\left(r_{n} e^{i \theta}\right) \geq \frac{1}{2}, \quad \text { for } e^{i \theta} \in E
$$

such an $r_{n}$ exists since $I_{n}$ is a union of finitely many arcs. Let $U_{n}^{*}$ be the harmonic conjugate of $U_{n}$ on the disk $\Delta$ which vanishes at 0 and set

$$
\varphi_{n}(z)=U_{n}\left(r_{n} z\right)+i U_{n}^{*}\left(r_{n} z\right), \quad z \in \bar{\Delta} .
$$

Since $\varphi_{n}$ is analytic on $\bar{\Delta}$ it is clear that $\varphi_{n} \in D$. We proceed to estimate $\left\|\varphi_{n}\right\|$. First,

$$
\hat{\varphi}_{n}(0)=\sigma_{n}(T) \ln 4=\hat{\sigma}_{n}(0) \ln 4 .
$$

Next, for $k>0$ and $h(t)=\ln 4 /\left|1-e^{i t}\right|$,

$$
\begin{aligned}
\hat{\varphi}_{n}(k) & =2 r_{n}^{k} \hat{U}_{n}(k)=2 r_{n}^{k} \widehat{h * \sigma_{n}}(k) \\
& =2 r_{n}^{k} \hat{h}(k) \hat{\sigma}_{n}(k)=\frac{r_{n}^{k} \hat{\sigma}_{n}(k)}{k} .
\end{aligned}
$$

Thus,

$$
\left\|\varphi_{n}\right\|^{2} \leq\left|\hat{\sigma}_{n}(0)\right|^{2} \log ^{2} 4+\sum_{k=1}^{\infty}(k+1) \frac{\left|\hat{\sigma}_{n}(k)\right|^{2}}{k^{2}} \leq 2 \operatorname{cap}\left(I_{n}\right),
$$

where we have used (4). It follows now that $\sum \varphi_{n}$ is in $D$ and

$$
\left\|\sum \varphi_{n}\right\| \leq \sqrt{2} \sum \operatorname{cap}\left(I_{n}\right)^{1 / 2}<\infty .
$$

Furthermore, since $0<\operatorname{Re} \varphi_{n}(z)<1$ for $z \in \Delta$, we have that

$$
f(z)=\exp \left(-\sum \varphi_{n}(z)\right)
$$

is in $H^{\infty}$; in fact, $\|f\|_{\infty} \leq 1$.

We claim that

(5) $f \in D$;

(6) $[f]=D$, i.e. $f$ is cyclic in $D$;

(7) $f$ is continuous on $\bar{\Delta}$;

(8) $Z(f)=E$;

and thus Theorem $B$ is proved.

To prove (5) observe that $f^{\prime}(z)=f(z) \sum \varphi_{n}^{\prime}(z)$. Since $f \in H^{\infty}$ and $\sum \varphi_{n} \in D$ it follows easily that $f \in D$. 
To verify (6) let $F_{N}(z)=\exp \left(-\sum_{n>N} \varphi_{n}(z)\right)$. Since $\exp \left(\varphi_{n}\right)$ is analytic on a neighborhood of $\bar{\Delta}$ one shows without difficulty that

$$
F_{N}=\prod_{n=1}^{N} \exp \left(\varphi_{n}\right) \cdot f \in[f] \text {. }
$$

It is easily checked that $\lim _{N \rightarrow \infty}\left\|F_{N}-1\right\|=0$. Thus $1 \in[f]$ and it follows that $f$ is cyclic.

To check (7) and (8) we show first that

$$
v(z)=\sum_{n=1}^{\infty} \operatorname{Re}\left(\varphi_{n}(z)\right)=\sum_{n=1}^{\infty} U_{n}\left(r_{n} z\right)
$$

is continuous in the extended sense on $\bar{\Delta}$. If $z_{0} \in \bar{\Delta}$ and is a positive distance from $E$, i.e. dist $\left[z_{0}, E\right]>0$, then it follows that for $n$ sufficiently large

$$
\operatorname{dist}\left[r_{n} z, I_{n}\right] \geq \delta>0
$$

for all points $z$ in some open ball centered at $z_{0}$. Thus,

$$
U_{n}(z)=\int_{I_{n}} \log \frac{4}{\mid r_{n} z-e^{i \theta \mid}} d \sigma_{n}(\theta) \leq\left(\log \frac{4}{\delta}\right)\left\|\sigma_{n}\right\|=\left(\log \frac{4}{\delta}\right) \operatorname{cap}\left(I_{n}\right) .
$$

The Weierstrass $M$-test implies that $v$ is continuous in a neighborhood of $z_{0}$.

Next, for $z_{0} \in E$ it follows from the choice of $r_{n}$ that $v\left(z_{0}\right)=\infty$. Since $\operatorname{Re} \varphi_{n}$ is continuous at $z_{0}$ and $\operatorname{Re} \varphi_{n}\left(z_{0}\right) \geq \frac{1}{2}$, there is a ball $B\left(z_{0}, \varepsilon\right)$ centered at $z_{0}$ of radius $\varepsilon$ such that $z \in \bar{\Delta} \cap B\left(z_{0}, \varepsilon\right)$ implies $\operatorname{Re} \varphi_{n}(z) \geq \frac{1}{4}$ for $n=1,2, \ldots, N$, where $N$ has been chosen beforehand. Thus $v(z) \geq N / 4$ for $\left|z-z_{0}\right|<\varepsilon$. This shows that

$$
\lim _{z \rightarrow z_{0}} v(z)=\infty, \quad z \in \bar{\Delta}
$$

that is, $v$ is continuous in the extended sense.

The calculations above show that $|f|=e^{-v}$ is continuous on $\bar{\Delta}$ and that $Z(f)=$ $E$. To show $f$ is continuous on $\bar{\Delta}$ we need therefore only check continuity at $e^{i t} \in T \backslash E$. Let $\gamma_{t}$ be the line segment from 0 to $r_{n} e^{i t}$ and let $\partial / \partial n$ denote the outer normal derivative. Then

$$
\begin{aligned}
U_{n}^{*}\left(r_{n} e^{i t}\right) & =\int_{\gamma_{t}} \frac{\partial U_{n}(z)}{\partial n}|d z| \\
& =\int_{\gamma_{t}}\left[\frac{\partial}{\partial n} \int_{I_{n}} \log \frac{4}{\mid z-e^{i \theta \mid}} d \sigma_{n}(\theta)\right]|d z|
\end{aligned}
$$

and

$$
\left|U_{n}^{*}\left(r_{n} e^{i t}\right)\right| \leq \int_{\gamma_{t}} \int_{I_{n}} \frac{c}{\mid z-e^{i \theta \mid}} d \sigma_{n}(\theta)|d z| \leq \frac{2 c\left\|\sigma_{n}\right\|}{\operatorname{dist}\left[e^{i t}, E\right]}
$$

for $n$ sufficiently large. Since $\sum\left\|\sigma_{n}\right\|<\infty$ it follows that $\sum U_{n}^{*}$ is continuous at $e^{i t} \in T \backslash E$ and the proof is complete.

We wish to make two remarks:

(1) A modification of the above proof of cyclicity can yield that Carleson's original examples (see [2, Theorem 4]) are cyclic in $D$. Of course, these examples may not necessarily be continuous in $\bar{\Delta}$. 
(2) If $D_{\alpha}, 0<\alpha<1$, is the Hilbert space of analytic functions for which $\|f\|^{2}=\sum_{n=0}^{\infty}(n+1)^{\alpha}|\hat{f}(n)|^{2}$ is finite, then by using a suitable Bessel capacity instead of logarithmic capacity, one can prove theorems for $D_{\alpha}$ corresponding to [1, Theorem 5] and Theorem B of this note.

\section{REFERENCES}

1. L. Brown and A. L. Shields, Cyclic vectors in the Dirichlet space, Trans. Amer. Math. Soc. 285 (1984), 269-304.

2. L. Carleson, Sets of uniqueness for functions regular in the unit circle, Acta Math. 87 (1952), $325-345$.

3. __ Selected problems on exceptional sets, Van Nostrand, Princeton, N.J., 1967.

4. J.-P. Kahane and R. Salem, Ensembles parfaits et śeries trigonométriques, Actualités Sci. Indust., no. 1301, Hermann, Paris, 1963.

Department of Mathematics, Wayne State University, Detroit, Michigan 48202 